\title{
ANÁLISIS DE LAS CREENCIAS DE ALGUNOS DOCENTES DE EDUCACIÓN INICIAL CON REFERENCIA AL ENSEÑAR
}

Gabriela Pena*

Andrea Viña**

\section{Introducción}

Las autoras de este informe, maestras de Educación Inicial con cinco años de formación académica, y formación específica en el área, percibimos un vacío respecto de nuestra formación en Didáctica, comprendida esta como teoría de la enseñanza. Este hecho adquiere otras significaciones más profundas cuando constatamos que en el diseño de Educación Inicial se encuentran explicitadas las fuentes que hacen referencia a las Ciencias de la Educación, pero no hay mención expresa de la Didáctica, como una de aquellas donde el maestro pueda acudir para profundizar sobre su ser docente. Entendemos que reflexionar sobre estos temas, puede viabilizar nuevas maneras de comprender el oficio de enseñante y por lo tanto, llevar al encuentro de nuevas maneras de enseñar.

En nuestro trabajo cotidiano vemos que, como maestras, pensamos con facilidad propuestas de aprendizaje, en cambio la dificultad aparece a la hora de situarnos en nuestro rol de enseñantes. La incidencia de esta dificultad hoy más clara para nosotros, comenzó a tomar cuerpo cuando nuestro marco conceptual en relación con la Didáctica empezó a cambiar y cuando descubrimos que en las actuales concepciones de enseñanza y de aprendizaje se jerarquiza la intención del enseñante. En momentos de hondos cuestionamientos conceptuales acerca de los procesos de enseñanza y de los procesos de aprendizaje; reflexionar sobre estos aspectos puede ser muy importante ya que nos enfrenta a revisar y redefinir temas tan trascendentes en nuestra vida profesional como son: la Didáctica, ¿teoría de la enseñanza o metodología educativa?; rol docente: ¿cómo y para qué?; la relación intención e intervención; ¿determinante o no de nuevas formas de enseñar?

Nuestro propósito es entonces explorar las creencias que los docentes de Educación Inicial(es decir, maestros que atienden niños entre tres y cinco años) tienen sobre su rol de enseñantes, para tratar de visualizar, como decíamos en párrafos anteriores, las concepciones que ellos manejan acerca de la didáctica que sustenta sus prácticas, y de esta manera qué intencionalidad le otorgan a las mismas.

Creemos necesario explicitar, que al poner el énfasis en los docentes y en sus creencias, lo hacemos en el entendido de que es la dimensión humana de la educación lo que no podemos dejar de considerar. Por tanto, lo que los docentes sienten, creen y piensan es crucial, ya que la acción de cada sujeto sólo puede comprenderse en términos de su propio marco de significados, determinándose así, la intencionalidad en su tarea. Para guiar este proceso nos planteamos dos interrogantes que orientan nuestro trabajo: ¿Qué creen los docentes acerca del enseñar en Educación Inicial? ¿Cómo entienden ellos desde lo didáctico, su intervención? 


\section{Encuadre teórico}

Teniendo en cuenta los aspectos señalados en la introducción de este informe es que creemos importante definir en el marco teórico, algunos conceptos que desarrollaremos a continuación por su pertinencia para la investigación.

Como señalamos en la introducción, creemos que el concepto de enseñanza es clave para nuestra investigación y la comprendemos como la actividad propia que da esencia y define el rol del maestro. La consideramos como una práctica esencialmente reflexiva y también, como una forma de investigación educativa ${ }^{1}$, que permite al docente ir elaborando sus procesos de construcción a la hora de instrumentar sus prácticas, por medio de su deliberación crítica. Estos aspectos nos parecen relevantes a la hora de ejercer la profesionalidad docente.

Nos resulta interesante profundizar en el concepto de enseñanza y es por ello que nos permitimos discrepar con autores como Zabalza quien sostiene que el profesor es concebido: ..."como profesional del currículum"... (1996:16). A partir de lecturas que hemos realizado y de nuestros años de experiencia en Educación Inicial, creemos que es importante que el maestro en este nivel, como en todo el sistema escolar, deje de considerar que la enseñanza es un medio que lleva a cumplir un programa; por el contrario, deberá poner el desarrollo de la persona en un primer plano y convertir esto en su preocupación y ocupación constante como enseñante, ya que nos interesa poner énfasis en procesos de enseñanza que hacen referencia a entender la actuación profesional desde una dimensión intelectual y ética y no solamente como una forma técnico instrumental.

Concebimos la enseñanza desde un enfoque multifactorial, por el cual, además de establecerse relaciones asimétricas entre quien enseña y quien aprende, como bien señala Feldman, se supone: ..."una situación inicial asimétrica con respecto al conocimiento y el establecimiento de una relación que permita un cambio en esa situación mediante la obtención, por parte de quien no lo tiene, de aquello que no poseía inicialmente. Es rasgo central de este concepto de enseñanza el compromiso de dos personas, una que posee algún conocimiento o habilidad y otra que carece de ella, en algún tipo de relación para que el primer sujeto traspase lo que sabe" (1999:17). Así mismo coexisten otras situaciones que se dan en el mismo momento, siendo estas partes constitutivas del propio proceso de enseñar. Hay pues enseñantes y hay aprendices; hay un saber, y un saber enseñado, historias personales, situaciones socio-políticoculturales, transposiciones y configuraciones así como también emociones, intenciones, deseos, de los sujetos comprometidos en la situación.

Freire, ilustra muy bien esta concepción cuando afirma en su libro: "Cartas a quien pretende enseñar"; ..."que el enseñar y el aprender se van dando de manera tal que por un lado, quien enseña aprende porque reconoce un conocimiento antes aprendido (...) El aprendizaje del educador al educar se verifica en la medida en que el educador humilde y abierto se encuentre permanentemente disponible para repensar lo pensado, revisar sus posiciones; en que busca involucrarse con la curiosidad del alumno y los diferentes caminos que ella lo hace recorrer"... "No obstante no debe significar en modo alguno que el educador se aventure a enseñar sin la competencia necesaria para hacerlo". (1994:28,29). Indudablemente, la imagen de aprendiz que un maestro da a sus alumnos es sumamente significativa a la hora de incorporar modelos de aprendizaje permanente, que trasciende la edad y los roles y transforman el aprendizaje en una vivencia vital para todo ser humano.

Al transcribir estas ideas sentimos la influencia de nuestra biografía escolar previa, así como la influencia que tienen los procesos de formación académica y de socialización laboral y es por esto que hacemos especial mención a los modelos que como aprendices recibimos de nuestros maestros y profesores, los que interiorizamos, y los que no. Preguntarnos qué cosas conciernen específicamente a nuestro rol, nos lleva poco a poco a darnos cuenta que encontramos aspectos de nuestro que hacer que oscilan entre ser guía, escuchar las necesidades de los niños, "¿enseñar en Educación Inicial?" Las respuestas no son únicas, son contradictorias, confusas y muchas veces personales. Estas vivencias las compartimos con 
nuestras compañeras y lentamente, se hace cada vez mayor la necesidad de cuestionar nuestras propias teorías, confrontar supuestos, conectarse con el conocimiento desde otro lugar y desarrollar nuestra autonomía de pensamiento y de acción.

Vemos que profundizar en el ejercicio de poder verbalizar nuestros propios supuestos, experiencias y puntos de vista sometiéndolos a la crítica metódica, es la llave del cambio, puntapié inicial de este trabajo.

Remarcamos entonces, que es función inherente al docente el enseñar, ya que creemos que es este un aspecto intrínseco de su rol: rol profesional que trasciende la transmisión de conocimientos y que para ser tal, debe nutrirse como decíamos antes, de una reflexión permanente. El enseñante aprende del propio proceso del enseñar en el que por supuesto estarán implicado los aprendices. Desde nuestra experiencia como maestras de Educación Inicial, hemos ido descubriendo que el término enseñar implica no solamente atender procesos cognitivos, sino también aquellos otros, que tienen que ver con el proceso de socialización que el niño está viviendo en esta etapa como eje estructurador de su personalidad, llevando todos y cada uno de ellos al plano de la reflexión, para desde allí determinar la intención que tenemos como enseñantes a la hora de planificar nuestras prácticas. El rol docente no se reduce a poseer destrezas o conocimientos seguros que surgen de su propia autoridad científica; creemos en él como una acción reflexiva. Aparece entonces la necesidad de establecer una unión dialógica entre las prácticas y las teorías, ya que somos conscientes de que la disociación entre la teoría y la práctica desvirtúa el propio proceso de enseñanza, haciéndoselo perder su propio carácter educativo truncando el vínculo enriquecedor entre el conocimiento y la acción. Si este vínculo entre teoría y práctica se va construyendo, contaremos con maestros más sólidos que puedan explicar las razones de sus actos; estos aspectos nos ayudan a reconceptualizar las ideas que poseemos acerca de la tarea de enseñar, así como la posibilidad de repensar nuevas maneras y formas de enseñar. Creemos que todo esfuerzo de unión dialógica entre teoría y práctica lleva a lo que Gimeno Sacristán y Pérez Gómez definen como "una acción informada y reflexiva, a la vez que un conocimiento educativo comprometido con opciones de valor y depurado en las tensiones y resistencias de las prácticas" (1994:14). Ahondar en el rol de enseñante supone crecer en la capacidad de reflexionar sobre la acción; es así que el rol docente se torna más profesional y sólido.

Como decíamos unas líneas más arriba, la educación no flota en el vacío ni tampoco está estancada, al decir de Giroux "Si queremos tomar en serio la enseñanza, las escuelas deben ser espacios donde las relaciones sociales democráticas formen parte de las experiencias vividas en cada uno de nosotros" (1990:49). La permanente adecuación a los contextos culturales y los proyectos políticos, la constante elaboración conceptual y las intervenciones acordes en cada situación con los sujetos y con los valores conscientemente asumidos, requieren del docente, un fuerte compromiso, así como la construcción de una autonomía profesional y condiciones institucionales para expandirlas. Muchas veces, las relaciones de poder político e institucionales son contrarias a estas intenciones. La individualidad de cada docente se construye en términos de mediaciones pues existe una estrecha relación entre construcciones personales, estructuras políticas, estructuras sociales e institucionales. Por otro lado, las instituciones poseen siempre la doble naturaleza de posibilitar la acción colectiva por un lado y tornar imposible determinadas formas de acción por el otro.

Por su parte Litwin, sostiene que la didáctica debe ser entendida como: ..."teoría acerca de las prácticas de enseñanza significadas en los contextos socio-históricos en que se inscriben" (1997:93). Creemos que las prácticas de enseñanza presuponen una identificación ideológica que hace que los docentes estructuren ese campo de una manera particular y realicen un recorte personal, fruto de sus historias, perspectivas y también limitaciones. Los docentes llevan a cabo las prácticas en contextos que las significan y en donde se visualizan planificaciones, rutinas y actividades que dan cuenta de este entramado complejo. 
Desde esta perspectiva entonces, nuestra construcción conceptual nos va acercando hacia una enseñanza que tiene una dimensión instrumental ${ }^{2}$ pero que también tiene una dimensión ético política; y alejándonos de una didáctica sinónimo de método donde las propuestas significaban el refuerzo de la certeza metódica, basadas en el "objetivismo", la "neutralidad política", la "eliminación simbólica del sujeto" y los "hechos de consciencia". Contrariamente, a esta postura Díaz Barriga tiene la convicción, de que la didáctica es una disciplina: ..."teórica, histórica y política". Estas tres dimensiones nos hablan de una diferencia significativa en la comprensión de las distintas prácticas de los docentes. Mientras que en el primer modelo, (el tecnicista) la acción es entendida como la realización de un plan trazado previamente con la racionalización de los instrumentos que utiliza persiguiendo la eficiencia y el éxito de sus realizaciones. Feldman identifica esta forma de entender la acción como una modalidad técnica. El autor hace referencia a la techne, como: ... "la forma de conocimiento y de razonamiento que correspondía a la acción productiva" (1991:49), en contraposición al modelo tecnicista, nace otra alternativa posible, denominada por él como modalidades prácticas. Estas hacen referencia a la phronesis, consiste en una forma de razonamiento práctico o deliberativo. La acción práctica estaría guiada por la prudencia que se impone en el análisis, de una forma de reflexión y deliberación de los hechos. Feldman plantea que: "El objetivo de la modalidad práctica es la toma de una decisión que guíe la acción. Estas decisiones tienen siempre un valor relativo a la situación y sólo pueden ser juzgadas por la comparación a otras alternativas" (1999:56,57).

Compartimos la idea de asumir desde nuestro rol la modalidad práctica ya que entendemos que se ajusta a nuestras concepciones. En esta forma de entender el acto de enseñar es que creemos que la intención pedagógica surge, a través de un proceso deliberativo, por medio del cual el docente reflexiona de qué manera llevará a cabo los medios para alcanzar los fines es decir cómo instrumentará sus modalidades prácticas. La forma en que cada uno de nosotros como docentes instrumentamos la práctica de nuestra profesión, está íntimamente relacionada con la concepción de "construcción metodológica" que Edelstein (en Litwin, 1997) desarrolla, planteando que ésta es relativa, no absoluta, contextualizada y singular. Esto implica tener en cuenta al sujeto que aprende y al objeto con su lógica particular, tornándose imprescindible asumir lo que Díaz Barriga plantea cuando afirma que el docente deberá: "(...)desconstruir ciertas estructuras producidas para ser apropiadas, construidas o reconstruidas por el sujeto de aprendizaje" (1985:81). Es válido desde esta perspectiva donde nos hemos situado pensar que los docentes de Educación Inicial, deberán transitar este camino de construcción teórico-práctica, por medio de procesos deliberativos que esclarezcan su intencionalidad a través de una actitud reflexiva a la hora de re-pensar su "oficio de enseñar"3.

Muchas veces, a lo largo de nuestro día a día en el aula nos vemos asaltadas por la necesidad de esclarecer si estamos llevando adelante una "buena enseñanza" (Davini,1995); responder a esta interrogante es para nosotras muchas veces un problema ético que incluye cuestiones de ideología y de compromiso con la problemática sociocultural de nuestros alumnos concretos. Hoy sabemos que la reflexión nos permite resignificar ideas, asumir nuevas implicancias o considerar otras alternativas, sintiéndonos muchas veces que más que de un acto de descubrimiento nos encontramos en un acto de invención, buscando nuevas alternativas disparadas por el proceso reflexivo, tratando de contestarnos como antes planteábamos, ¿Qué conviene hacer?, ¿Qué es posible?, ¿Cómo hacerlo?, desde lo educativo. Estamos transitando en este momento de nuestra vida profesional por un camino de estructuración y nueva construcción conceptual; pero ya no con la angustia que muchas veces esta sensación de vacío profesional nos provocaba, sino por el contrario, con la riqueza de saber que estamos en este proceso creativo, dinámico y humano, en tanto personal, apostando al perfeccionamiento de nuestra tarea.

Nos parece importante aclarar que si bien hablamos unas líneas más arriba de un proceso personal, no lo entendemos a este como un camino en solitario, sino todo lo contrario: como un andar que el docente realiza compartiendo con otros, superando el aislamiento que muchas veces vive en su actividad profesional. Al respecto Elliot plantea que: "Mediante la reflexión y el diálogo es posible progresar en el desarrollo de formas compartidas de comprensión de los conceptos éticos y de los dilemas contradictorios 
de la práctica" (1983:17). Creemos que es de hondo valor detenernos a revisar nuestras prácticas educativas, debatiendo sus supuestos, estrategias y consecuencias, siempre orientándonos a favorecer el desarrollo de una enseñanza para la comprensión. Creemos que es desde aquí que se analizan y sostienen los enfoques didácticos. Valoramos sin duda como antes habíamos dejado establecido, la importancia de pensar la enseñanza desde ámbitos de confrontación y debate, con la finalidad de lograr un saber teórico práctico que nace de la investigación sistematizada de los propios actores -los maestros- por medio de procesos deliberativos.

Todo este desarrollo nos lleva a concluir que el ser docente implica hacerse profesional de la enseñanza. Imbernón, hace importantes aportes al respecto, él sostiene que: "Se trata de ver la formación como un aprendizaje constante, acercando ésta al desarrollo de actividades profesionales y a la práctica profesional y desde ella (...) se establece un proceso dinámico que supera los componentes técnicos y operativos impuestos desde arriba sin tener en cuenta el colectivo y las situaciones problemáticas de la práctica del profesor, permitiendo considerar la práctica de la enseñanza como una profesión dinámica" (1997:10). Nos preguntamos ¿Qué caminos de reflexión deliberativa recorren hoy los maestros de Educación Inicial? ¿Cuánto hay en ellos de profesionalismo, tal y cómo lo plantean los autores citados? Creemos que una forma de contestar a estas interrogantes es indagar en las creencias de los docentes respecto de sus prácticas y del rol que ellos creen deben ocupar como profesionales de la enseñanza.

Nuestro campo de investigación se enmarca en el Nivel Inicial del Sistema Formal de Educación, por tal motivo nos parece ajustado describir y señalar algunas peculiaridades de este sector de la enseñanza en el cual trabajamos. En Educación Inicial, por sus características históricas concretas, la situación del profesionalismo es difusa con un perfil, una identidad todavía en proceso de construcción. Históricamente, la Educación Inicial tiene las primeras menciones hacia fines del siglo pasado con Varela y la maestra E. Compte y Riquet. En 1892, se funda el primer jardín de infantes del país con el método froebeliano y los principios varelianos. No es hasta mediados del siglo siguiente, que con la fundación del segundo jardín de infantes se expande la educación preescolar en nuestro país. A nivel social, en el período comprendido entre esos años, se produce la salida a nivel laboral de la mujer. Con ello, la necesidad de un lugar donde dejar a sus hijos. La expansión de las llamadas guarderías a partir de los años 60 es un hecho que nos hace preguntarnos: - ¿en qué momento empieza a conformarse la identidad propia de Educación Inicial? Seguramente la respuesta la encontramos no muchos años atrás, pues recién en el año 1996 la Inspección Nacional de Preescolares cambia su denominación y pasa a llamarse Inspección Nacional de Educación Inicial. Creemos que esta modificación no pretende ser superficial sino que denota la consolidación de un subsistema con características propias y en donde los docentes también tienen una intención que le es propia, tratando de determinar, como plantea Imbernón, una cultura profesional propia de este subsistema.

Hemos querido dejar para el final del desarrollo del marco conceptual, la definición de lo que se entiende por el término creencia, en esta investigación. Apoyándonos en Merieu diremos que las creencias están en íntima relación con el sistema de representaciones, que el autor, define como: "Conjunto de concepciones (imágenes, metáforas, modelos) que permiten organizar los datos de la percepción y pretenden dar cuenta de lo "real". Un sujeto dispone siempre de un sistema de representaciones de una realidad". (1992:211). Este sistema de representaciones se impregna en todo el "ser" y "hacer" docente sellando cada práctica áulica con las características propias de cada docente; esto supone pues, indagar el mundo de la subjetividad, que es desafiante, pero a la vez necesario; en esta búsqueda de conectarnos con las concepciones, modalidades, sentimientos, acciones de nuestros colegas para acercarnos a comprender su realidad, en el momento que estructuran y accionan sus estrategias de enseñanza, determinadas sin lugar a duda por aquello que ellos creen. 
Desde un enfoque interpretativo de investigación sentimos que reflexionar sobre los elementos expuestos puede llegar a ser un valioso instrumento para ir transformando el "hecho educativo" y así lograr como maestros construir y reconstruir, nuestras prácticas, recreando instancias educativas que vayan más allá de las prácticas de aula que alguna vez experimentamos. Sentimos que esta mirada detenida y cuidadosa en estos asuntos, nos aporta la relevante riqueza de ahondar en la figura misma del maestro, y así poder ir comprendiendo su papel de profesional de la enseñanza, como propio aprendiz de la sociedad y de la cultura". Al decir de Pérez Gómez: "La actitud y el concepto que se tiene de sí mismo como profesional no será extraño que se proyecte en las inseguridades, en la tolerancia y en la actitud epistemológica ante la cultura que colabora en que otros descubran y en las técnicas pedagógicas por medio de las que lo intente (1994:16).

\section{Diseño metodológico}

Con relación a lo metodológico, diremos que nos parece adecuado un modelo de investigación cualitativa que contemple las peculiaridades del fenómeno que es objeto de estudio. Así como consideramos que el modelo de investigación debe estar determinado por el problema de investigación, creemos que es pertinente que los instrumentos a utilizar estén acordes con el modelo y por tanto, con el problema a investigar.

Hemos seleccionado la entrevista como el instrumento a utilizar, en el entendido de que es aquel que nos permite acercarnos más al problema de investigación. Su objetivo prioritario al decir de Pérez Gómez "(...) es captar las representaciones e impresiones subjetivas más o menos elaboradas de los participantes" (1994: 27). Optar por la entrevista, nos ha permitido llegar a la información por medio de los propios agentes; como plantea Geertz "Los estudios basados en entrevistas tratan que los lectores tengan la sensación "están en la piel" de los informantes y vean las cosas desde el punto de vista de ellos. De modo que la investigación cualitativa proporciona una "descripción íntima de la vida social". (1983:37). Estos aspectos nos permitieron comprender mejor qué opinan, creen, sienten, piensan las personas involucradas, los docentes. Además en el contacto directo pudimos registramos: silencios, gestos, estados emocionales, etc. que cobraron mucha significación en el momento de analizar la información de las entrevistas.

Nos valimos además del análisis de documentos, cuyos contenidos los constituyen las planificaciones de algunos de los docentes entrevistados como fuente de información, para adquirir conocimientos de las personas que las redactan y así poder verificar qué tipo de vínculo establecen entre lo que creen y registran. Al decir de Taylor y Bogdan: "Estos documentos permiten comprender las perspectivas, los supuestos, las preocupaciones y actividades de quienes los producen". (1998:149). Debido a su "naturaleza subjetiva" la información obtenida ha llegado a ser de gran valor a la hora de saber acerca de las creencias de los docentes, para lograr describir con más rigor lo analizado o arribar a las conceptualizaciones que se manejan.

Contamos por otra parte en todo el proceso de esta investigación con la validación externa de un especialista reconocido en Didáctica, que fue acompañando, revisando y orientando nuestra tarea. Confrontamos con él la información que fuimos recabando y gracias al respaldo que él iba dando a nuestra tarea nos aproximamos a algunas reflexiones que aparecen planteadas al final de este trabajo.

Llevar a cabo una entrevista requiere de ciertos cuidados que atendimos adecuadamente. Algunos aspectos que tuvimos en cuenta, en el momento de operativizarla fueron: en primer lugar, entrevistar a aquellas personas que así lo deseen; la entrevista se efectúa en un ambiente adecuado que favorezca la concentración del entrevistado, de esta manera estamos favoreciendo al máximo la producción del discurso, logrando comunicación y empatía. En segundo lugar, establecer el "Contrato Inicial de Comunicación", por el cual explicamos en qué consiste la entrevista y explicitamos que su aporte es anónimo en la 
investigación. Solicitamos autorización para grabar la realización de la entrevista como también, una vez desgrabada entregamos el texto para corroborar y reafirmar que se encuentran escritas las ideas que el entrevistado quiso manifestar, dándosele así, la oportunidad de ampliar, rectificar o desechar lo que él crea conveniente.

Aquí nos interesa remarcar el interés que tenemos en este trabajo, de conocer sobre las creencias de los docentes; es por esta razón que le damos importancia al hecho de que el entrevistado pueda volcar en el momento de la entrevista -y en esta segunda instancia-, su sentir y su parecer, sobre el hecho de enseñar. No se efectúan por parte del entrevistador ninguna señal o comentario que dejen traslucir sus puntos de vista sobre lo que escucha. Nos limitamos a oir y entender el discurso del entrevistado.

La entrevista fue semi-pautada; de esta manera, nos aseguramos un marco común que nos permite llegar al objetivo propuesto. Esta técnica no es sencilla y requiere del entrevistador experiencia que nosotras no tenemos. Por otra parte, al ser semi-pautada, dejamos un margen de apertura que no limite aquellos giros propios de cada una de las situaciones, en las cuales el entrevistado considere pertinente profundizar. Esto se relaciona directamente con una de las características más destacadas de ese instrumento que es el de ser abierto. Las entrevistas fueron realizadas de un entrevistado y de un entrevistador por vez, en el entendido de que es esta la mejor manera de lograr una comunicación fluida.

Nuestro tema de investigación, presenta una clara realidad polisémica, una dialéctica entre lo observable y el significado latente. Pensamos que el mundo de las creencias fluctúa permanentemente entre esta dialéctica significado - significante; realidad-subjetividad. Utilizamos entonces en la metodología procedimientos que nos permiten comprender el significado, las percepciones respecto de las creencias (que de alguna forma nos dicen mucho de su rol) inmersas en el contexto en que se producen y se manifiestan, sin aislarlas.

Decimos entonces, que este enfoque es claramente interpretativo, busca comprender la forma en que los individuos conciben el hecho educativo del cual forman parte. Esto tiene que ver con las conceptualizaciones que hacen tanto a nivel individual como grupal, en el entendido de que una y otra se retroalimentan y los significados se modifican. Al decir de Pérez Gómez: "No existe una única realidad en el ámbito de lo social en general y de lo educativo en particular, sino múltiples realidades que se complementan mutuamente (...). Por otra parte las representaciones subjetivas de los hechos son tan importantes como los hechos mismos (...)" (1994:119)

Dado que la investigación tiene un enfoque cualitativo e interpretativo, el objetivo no es la predicción ni el control de variables sino la comprensión de los fenómenos. Cada situación no es independiente de su contexto, entonces la comprensión de los significados no puede realizarse con independencia del mismo. El propósito entonces fue sumergirnos por medio de la metodología, entre otras cosas, en la complejidad de las creencias, para indagar sobre ellas con la libertad y la flexibilidad que requieren, elaborando descripciones y abstracciones de los datos.

Los sujetos de investigación fueron Maestros de Educación Inicial, titulados como tales, que cumplen tareas de docencia directa egresados de diferentes planes de estudio. Puntualizamos aquí, que el grupo de informantes de esta investigación lo constituyeron cinco maestros; la selección fue realizada en forma no probabilística e intencional, teniendo en cuenta el problema que estuvimos investigando. A los entrevistados llegamos aplicando la técnica de la "bola de nieve" de la siguiente manera: las dos primeras son seleccionadas por nosotras y a ellas aplicamos el pretesto; luego, y en forma sucesiva nosotras le indicamos el plan de estudios y años de trabajo que debían tener los docentes y ellos nos contactaron con un nuevo entrevistado y así sucesivamente. Cabe aclarar que en los casos en que la entrevista no pudo realizarse, volvíamos a la persona que nos había contactado y le solicitábamos un nuevo informante con las mismas características. Pensamos que podrían haber un sin fin de factores que pudieran estar incidiendo en las creencias de los maestros (historias personales, edad, años de trabajo, órbita laboral pública o privada, cursos de actualización); pero ante la necesidad de acotar el campo en esta oportunidad, no pudimos abordarlos en su totalidad. 
En relación con los entrevistados, podemos decir que estos pertenecen tanto a la órbita pública como privada, de ambos sexos y con una variada gama de años de trabajo ya que entrevistamos a maestros en el inicio de su carrera, en medio de la misma y otros próximos a jubilarse.

Es evidente que toda investigación se realiza desde una plataforma o matriz conceptual, un paradigma (Kuhn, 1975) que define las características del objeto de investigación; el tipo de problemas a plantear y resolver, la propia esencia de los procesos de investigación, las estrategias, técnicas e instrumentos que se consideran más adecuados y los criterios de validación y legitimación del conocimiento producido. Aclaramos también que nuestro juicio, nuestra sensibilidad profesional como investigadores son insustituibles instrumentos para captar la complejidad y polisemia de los fenómenos educativos, así como para adaptarnos con flexibilidad a los cambios y a la aparición de acontecimientos imprevistos y anómalos.

\section{Recolección de la información.}

Durante los meses de octubre y noviembre nos abocamos a la recolección de la información que nos habíamos propuesto obtener de parte de nuestros colegas. Para ello, lo primero que implementamos fue una pauta de entrevista con la finalidad de neutralizar de alguna manera las diferencias que inevitablemente surgen al ser dos los entrevistadores. Por otro lado, dada nuestra inexperiencia en el uso de la técnica, consideramos que tener esta pauta nos brindaba tranquilidad en el uso de la misma y nos encauzaba a encontrar la información que buscábamos.

La pauta de entrevista que elaboramos fue la siguiente:

1- ¿Cuál es el plan de estudios que has cursado? Podrías comentarme si tu formación o estudios posteriores han tenido peso en tu trabajo cotidiano. Hay algún modelo o corriente a la que te sientas apegada desde aquel momento. ¿ Has cambiado?

2- El trabajo que realizas es multifacético. ¿Qué aspectos eliges para estructurar tu tarea? ¿En tu tarea de enseñar qué aspectos jerarquizas?

3- Me podrías contar alguna experiencia que consideres exitosa. ¿Qué te llevó a pensarla de esa manera? ¿A qué le atribuirías el éxito? ¿Por qué fue exitosa? ¿La diseñaste sola?

4- Me podrías contar alguna experiencia que consideres no exitosa. ¿Por qué? ¿Qué fue lo que desde tu punto de vista no salió bien?

5- ¿Piensas que tu planificación es importante?

6- ¿Cómo entiendes que debe ser tu intervención como docente?

7- ¿Cómo definirías tu tarea? (Luego ir paulatinamente planteando ideas como facilitador, guía, etc..)

8- ¿Cómo definirías tu propuesta de enseñanza?

9- ¿Qué es para ti la Didáctica? ¿Te animarías a darme una definición sobre la enseñanza en Educación Inicial?

10- ¿Quieres agregar algo más, algún comentario?

Una vez pronta la pauta de entrevista decidimos, aplicar dos entrevistas (una por cada entrevistador) a modo de pretest como forma de confirmar que la información que deseamos obtener era factible de ser encontrada por medio de esas preguntas. Afortunadamente esas entrevistas fueron exitosas y el cuestionario guía quedó incambiado para efectuar las entrevistas que aportaron los datos para nuestro trabajo. 
Nos parece interesante explicitar que la concreción de las entrevistas fue bastante difícil, ya que las y los maestros en primera instancia aceptaban realizar la entrevista y luego cuando llegaba el día acordado, surgían inconvenientes en forma reiterada habiendo sido imposible en algunos casos la concreción de las mismas. Nos vimos entonces obligadas a buscar otros docentes, a ser muy pacientes y agendar y re-agendar varias veces las mismas entrevistas. Suponemos que esto que se dio reiteradas veces y con casi todos los docentes nos podría estar hablando de una dificultad por parte de los mismos a someterse a la entrevista. Las razones pueden ser diversas, no pudiendo dejarse de considerar que el momento del año lectivo en el que nos encontramos al hacer las entrevistas, priman el cansancio y el estrés en casi todos los docentes. Por otro lado, creemos que el aislamiento en que generalmente trabajan los maestros así como la falta de reflexión sobre estos temas propios del "ser docentes" podrían estar poniendo en juego resistencias, que se accionan sobre todo a la hora de compartir con otro cosas que son "muy personales".

\section{Análisis de la información}

\subsection{Las entrevistas}

De la opinión de los docentes entrevistados surgió información que se focalizó hacia las preguntas de investigación que nos hemos formulado en este trabajo, como ya lo hemos señalado, indagamos sobre las creencias acerca del enseñar, el rol docente, las formas de intervención y las concepciones acerca de la didáctica.

Luego de varias lecturas hemos sistematizado esta información en tres categorías de análisis:

Categoría 1. Concepciones y creencias acerca del enseñar.

Categoría 2. Creencias acerca de la didáctica.

Categoría 3. Formas de intervención docente.

Nos pareció que esta posible categorización reflejaba por un lado, claramente lo que deseábamos averiguar y a su vez, nos daba la posibilidad de establecer subcategorías dentro de ellas mismas, ya que el análisis de la información nos enfrentó a un panorama de la realidad conceptual de los docentes que es polisémico. Por ejemplo, en la Categoría 1 Concepciones y creencias acerca del enseñar, en lo explicitado por el entrevistado número uno encontramos como subcategoría: crear hábitos, poner límites, crear buen ambiente de trabajo. Ser modelo, dar imagen, ser referente. Enseñanza es todo; es la construcción del conocimiento. Creemos que esta aclaración facilita la lectura del cuadro que presentamos a continuación.

\section{Categoría 1: Concepciones y creencias acerca del enseñar.}

Nos parece interesante mostrar que en esta categoría algunos docentes son partidarios de no enseñar y otros consideran que sí deben enseñar; si bien estas dos concepciones aparecen como posturas antagónicas muchos entrevistados a lo largo de la entrevista revelan en su discurso coexistencia de ambas posturas. Dentro del grupo de los maestros que en el momento de su discurso, plantean el hecho de que ellos "no enseñan" hay una clara evocación de enseñar, como llevar adelante un modelo tradicional, expositivo, frontal y en algunos casos coercitivo. Dan cuenta de esto, expresiones tales como:

"Antes el maestro enseñaba, ahora el maestro no enseña, digo, guía y ayuda, organiza lo que le traen..." (E3:1)

"...para mí es como una cosa conductista enseñar. Se le enseña a un perro que orine fuera de su casa. Eso me parece que es enseñanza en este momento, no, me gusta más la palabra educar, formar un ser integral". (E5:1) 


\section{Cuadro $N^{0} 1$}

\begin{tabular}{|c|c|c|c|}
\hline Entrevistas & $\begin{array}{l}\text { Concepciones y creencias } \\
\text { con respecto al enseñar y } \\
\text { rol docente }\end{array}$ & $\begin{array}{l}\text { Creencias acerca } \\
\text { de la Didáctica }\end{array}$ & $\begin{array}{l}\text { Formas de } \\
\text { intervención } \\
\text { docente }\end{array}$ \\
\hline Entrevistado 1 & $\begin{array}{l}\text { Básicamente crear hábitos poner } \\
\text { límites, crear buenos ambientes de } \\
\text { trabajo. Ser modelo, dar imagen, } \\
\text { ser referente. Enseñanza es todo, } \\
\text { es la construcción del } \\
\text { conocimiento. }\end{array}$ & $\begin{array}{l}\text { Es como trabajas para } \\
\text { atender realidades con lo } \\
\text { que tenes y los resultados a... } \\
\text { Es el } 90 \% \text { del éxito } \\
\text { como maestro. }\end{array}$ & $\begin{array}{l}\text { Buscar algo útil para los } \\
\text { alumnos. Trabajar con padres. } \\
\text { Brindar material informativo. } \\
\text { Contextualizar el hacer. } \\
\text { Sirve para reflexionar. } \\
\text { Evaluar constantemente. }\end{array}$ \\
\hline Entrevistado 2 & $\begin{array}{l}\text { Enseñar, es: llevar los } \\
\text { contenidos de lo teórico } \\
\text { a lo práctico. Cooperar, } \\
\text { respetarse y escuchar. Secuencia } \\
\text { de actividades que puedan hacer } \\
\text { que ese contenido sea vivenciado. } \\
\text { La enseñanza puede quedar } \\
\text { relegada. Rol es: organizar ideas } \\
\text { previas para elaborar propuestas, } \\
\text { ser modelo, buenos interventores, } \\
\text { contenedores y desestructuradores. }\end{array}$ & $\begin{array}{l}\text { "Es una disciplina, no sé } \\
\text { si es..." Es una herramienta } \\
\text { para llevar adelante las } \\
\text { actividades del aula. }\end{array}$ & $\begin{array}{l}\text { Es relación afectiva y vínculo } \\
\text { antes que nada. Propuestas } \\
\text { que interesan al niño. }\end{array}$ \\
\hline Entrevistado 3 & $\begin{array}{l}\text { "Yo no sé si enseño, lo que sé es } \\
\text { que ellos aprenden, pero si enseño } \\
\text { o no, no sé ni me interesa." "Antes } \\
\text { enseñaba ahora, no. Guío, ayudo a } \\
\text { a organizar". "El niño aprende más } \\
\text { solo, que del maestro". Rol ahora } \\
\text { es menos frontal. }\end{array}$ & $\begin{array}{l}\text { "Algo que vi hace mucho } \\
\text { tiempo ya no me acuerdo". }\end{array}$ & $\begin{array}{l}\text { Ellos hacen, tú guías. Mostrar } \\
\text { al niño lo que él no sabe. } \\
\text { Provocar conflicto, distinto a } \\
\text { posturas tradicionales. }\end{array}$ \\
\hline Entrevistado 4 & $\begin{array}{l}\text { Enseñante, no. Suena imperativo. } \\
\text { "...provocar procesos de } \\
\text { apropiación". "Me siento } \\
\text { ecléctica". }\end{array}$ & $\begin{array}{l}\text { Es la implementación de } \\
\text { temas teóricos que se llevan } \\
\text { a las prácticas. }\end{array}$ & $\begin{array}{l}\text { Mostrar formas de } \\
\text { relacionamiento, de } \\
\text { conviviencia. Formadora de } \\
\text { hábitos. Dar el ejemplo. } \\
\text { Expositiva, asocia a } \\
\text { conductista. "Trato de no } \\
\text { serlo porque está mal". }\end{array}$ \\
\hline Entrevistado 5 & $\begin{array}{l}\text { Freire, posición horizontal. Rol, de } \\
\text { diálogo, gusto por el ser humano. } \\
\text { Vocación como fundamental. } \\
\text { Maestro facilitador de procesos } \\
\text { de aprendizaje y de la autonomía. } \\
\text { No autoritario. Figura de referencia } \\
\text { afectiva para el niño. Enseñar es } \\
\text { algo que está en vos, que le querés } \\
\text { meter al otro. No me gusta. } \\
\text { Educación me gusta más, es a todo } \\
\text { un ser integral. Educación como } \\
\text { proceso democratizador. }\end{array}$ & $\begin{array}{l}\text { Es una metodología. Conjunto } \\
\text { de reglas para "enseñar". }\end{array}$ & $\begin{array}{l}\text { Relación afectiva para que } \\
\text { haya enseñanza. Conocer } \\
\text { bien con quien trabajas. } \\
\text { La comunicación. El juego } \\
\text { como gran hilo conductor. } \\
\text { Partir de ideas previas. } \\
\text { Proporcionar experiencias } \\
\text { de aprendizaje. }\end{array}$ \\
\hline
\end{tabular}


"Porque para mí, enseñar es algo que está en ti, que vos le querés meter al otro. Esta idea se me mete en la cabeza cuando escucho enseñanza". (E5:2)

"...yo te diría que enseñante no, porque me suena muy impositivo...facilitador en el sentido de tratar de poner a su disposición esas cosas que están ahí para que ellos puedan apropiárselas, ¿ no?. Este... pero muchas veces puede que caiga en la imposición..." (E4:1)

\section{Categoría 2: Creencias acerca de la Didáctica}

Dentro de esta categoría nos parece interesante destacar especialmente en los maestros jóvenes entrevistados, la clara demanda que aparece en relación con su formación dentro del Instituto Normal, especialmente con respecto a la Didáctica y a las prácticas. Todos hablan de una formación post-egreso, que poco pudo nutrirse de lo aprendido en el «Instituto Normal». Por otra parte, las respuestas obtenidas sobre las creencias de la Didáctica son variadas, podríamos decir tomando en cuenta el marco teórico explicitado en este informe, que las mismas entienden a la Didáctica como sinónimo de método. Las ideas encontradas en los entrevistados al respecto son:

"Y la Didáctica es metodología. Un conjunto de reglas ..." (E5: 4)

"Es la forma cómo vos le acercás el conocimiento al niño." (E1:2)

"...yo te puedo decir que en mi generación, en mi caso particular fue bastante mala en cuanto a la calidad de educación, de formación, yo salí del instituto, con poquísima base, yo creo que me hice en la experiencia, todo lo que sé, o casi todo lo aprendí después que salí del Instituto". (E4:3)

\section{Categoría 3: Formas de intervención docente ${ }^{4}$}

Algo muy interesante nos sucede cuando intentamos sistematizar la información que corresponde a esta categoría. Percibimos y vimos en las entrevistas, grandes dificultades para responder a las preguntas que apuntan a la intervención que el docente hace en su tarea de enseñar. Se constata esto, a través de silencios reiterados, pedidos de aclaración y solicitud de ayuda. Por otro lado, durante las entrevistas muchas veces se describen situaciones de clase donde aparece una clara intervención con una intención, pero esta no puede ser explicitada en forma consciente en todos los casos, cuando le pedimos que nos hablen de sus intervenciones. Para reflejar las situaciones en las cuales se perciben los silencios reiterados encontramos en el anexo 1 entrevistado 2:2tres espacios donde se registran silencios, posteriormente la entrevistada hace preguntas que la ayuden a orientarse en la respuesta a estas preguntas. En otra entrevista, en el anexo número1 apartado 4:4 encontramos expresiones tales como: "Así, en general decis... no sé... ahora como que se está yo que sé no sé... la tarea del docente de inicial es un poco distinta de la tarea del docente de común yo siempre puse como prioritario los aspectos de los hábitos porque creo que es una edad propicia para trabajar esos aspectos..."

"Y bueno yo creo que te lo dije a lo largo de la conversación. Para mí el maestro debe ser un guía mostrando a sus alumnos que hay cosas que él no sabe y que deben buscar juntos y promover lo intelectual en todo ¿no?" (E3:4)

"Si, facilitador. No como un maestro autoritario". (E5:5)

\subsection{Análisis del contenido de las planificaciones}

Para visualizar de forma clara y ágil los datos de contenidos de los registros a los que pudimos acceder, nos pareció adecuado sistematizar la información en el siguiente cuadro al que denominamos cuadro $\mathrm{N}^{\circ} 2$ que sintetiza por un lado, algunos datos del cuadro $\mathrm{N}^{\circ} 1$ e información tomada del registro de planificación de los docentes. Si bien nuestra intención fue acceder a los registros de todos los entrevistados esto no fue posible en dos casos debido a la negativa de los maestros, (estos maestros fueron los identificados con el número 4 y 5 respectivamente). 


\section{Cuadro $\mathbf{N}^{\circ} 2$}

\begin{tabular}{|c|c|c|c|}
\hline Entrevistas & $\begin{array}{l}\text { Creencias sobre el } \\
\text { enseñar y rol docente }\end{array}$ & $\begin{array}{l}\text { Formas de } \\
\text { intervención }\end{array}$ & Documentación \\
\hline Entrevistado 1 & $\begin{array}{l}\text { Crear hábitos y buen ambiente } \\
\text { de trabajo. Poner límites. } \\
\text { Ser modelo, dar imagen, ser } \\
\text { referente. } \\
\text { Enseñanza es todo, es la } \\
\text { construcción del conocimiento. }\end{array}$ & $\begin{array}{l}\text { Buscar algo útil para } \\
\text { los alumnos. } \\
\text { Trabajar con padres. } \\
\text { Contextualizar el hacer. } \\
\text { Sirve para reflexión. } \\
\text { Evaluación constante. }\end{array}$ & $\begin{array}{l}\text { Se registran: objetivos, } \\
\text { secuencias didácticas, } \\
\text { recursos didácticos, } \\
\text { estrategias y evaluación } \\
\text { de logros de los alumnos } \\
\text { (en blanco sin } \\
\text { completar). } \\
\text { Aparecen contenidos } \\
\text { conceptuales. } \\
\text { Se contemplan trabajos } \\
\text { con padres. } \\
\text { Aparecen contemplados } \\
\text { en los registros aspectos } \\
\text { afectivos emocionales. }\end{array}$ \\
\hline Entrevistado 2 & $\begin{array}{l}\text { Enseñar: Llevar contenidos de } \\
\text { lo teórico a lo práctico. } \\
\text { Cooperar, respetarse, escuchar. } \\
\text { Secuencias de actividades que } \\
\text { hacen que ese contenido } \\
\text { sea vivenciado. La enseñanza } \\
\text { puede quedar relegada. } \\
\text { Rol: organizar ideas previas, } \\
\text { ser modelo, buen interventor, } \\
\text { contenedor y desestructurador. }\end{array}$ & $\begin{array}{l}\text { Es relación afectiva y } \\
\text { vínculo antes que nada. } \\
\text { Propuestas que } \\
\text { interesan al niño. }\end{array}$ & $\begin{array}{l}\text { Aparece indagación } \\
\text { de ideas previas. } \\
\text { Se registran algunos } \\
\text { contenidos conceptuales. }\end{array}$ \\
\hline Entrevistado 3 & $\begin{array}{l}\text { "Yo no sé si enseño, lo que sé, } \\
\text { es que ellos aprenden, pero si } \\
\text { enseño o no, no sé ni } \\
\text { me interesa." "Antes enseñaba, } \\
\text { ahora no. Guío, ayudo a } \\
\text { organizar". "El niño aprende } \\
\text { más solo que del maestro". } \\
\text { Rol ahora, es menos frontal }\end{array}$ & $\begin{array}{l}\text { Ellos hacen, tú guías. } \\
\text { Mostrar al niño lo que } \\
\text { él no sabe. Provocar } \\
\text { conflicto, distinto } \\
\text { a posturas tradicionales. }\end{array}$ & $\begin{array}{l}\text { Evaluación de logros } \\
\text { prevista, pero en blanco, } \\
\text { sin completar. } \\
\text { Aparecen contenidos } \\
\text { conceptuales. } \\
\text { Se registra una salida } \\
\text { didáctica. } \\
\text { Se menciona } \\
\text { "modalidad talleres" }\end{array}$ \\
\hline
\end{tabular}

\section{Discusión}

Ya finalizando este informe, aparece nuevamente, la necesidad de replantearnos las inquietudes e interrogantes que desde el comienzo guiaron este proceso de investigación; explorar las creencias que los docentes de Educación Inicial tienen sobre su rol de enseñantes, y cómo entienden ellos desde lo didáctico su intervención. Durante este proceso, fuimos arribando a algunas razones que nos permitieron comprender aquella realidad que en el inicio de este trabajo, tanto nos movilizó.

Por un lado, nos encontramos con un fuerte grupo de maestros que manifiestan que no creen que su tarea en Educación Inicial sea la de enseñar, ya que ellos ven a "la enseñanza" como algo tradicional, expositivo y autoritario (al decir de los mismos entrevistados). Asimismo, en los momentos en que se habla de enseñanza se hace alusión a lo netamente cognitivo. La enseñanza vista como un proceso 
compartimentado no integrador de la persona. Frente a estas afirmaciones y en el intento de tratar de comprender "a resistencia" que estos docentes tienen al referirse al término de enseñar, podemos suponer que su biografía escolar, sumada a los mensajes contradictorios de las autoridades y de los programas vigentes, el gran vacío conceptual con relación "a las nuevas concepciones de didáctica" y cierta confusión en el manejo de los aportes de la psicología del aprendizaje (especialmente la teoría constructivista) son algunas de las explicaciones que podemos encontrar con relación a este tema.

Por otra parte, se reitera la idea que tienen los entrevistados sobre la falta de solidez académica con la que egresaron del Instituto Normal. La misma intenta ser suplida por la experiencia como solución a la inadecuada formación recibida: experiencia que como ellos nos dejan ver a lo largo de las entrevistas, no es enriquecida por la reflexión deliberativa. Nosotras creemos que la esencia misma de nuestra tarea nos impone la obligación de realizar una interpretación de los hechos, utilizando nuestro juicio crítico, como forma de hacer frente a los desafíos y construir nuestro quehacer docente sobre una conciencia más amplia y más crítica. Esto implica aspectos sobre los cuales los maestros también deberían ser formados. Nos queda la impresión, de que conciliar estos aspectos, algunos antagónicos, genera una tensión que impide a muchos docentes ubicarse desde su rol de enseñantes, ya que no se encuentran canales concretos de discusión, intercambio, de construcción compartida.

Nosotras mismas hemos experimentado esa sensación de incertidumbre al comienzo de este trabajo como maestras de Educación Inicial. Pensar nuestra tarea desde el rol de enseñantes nos llevó a ahondar en muchas revisiones sobre lo que poseemos internamente, como aprendices primero y posteriormente como enseñantes. Es aquí donde lo que creemos respecto a nuestra tarea es importante poderlo explicitar, para poderlo analizar en confrontación con otras posturas y evidencias e investigaciones que se encuentren sobre ella. El ser docente pasa por reflexionar sobre nuestra labor como enseñantes y nos parece muy oportuno para ejemplificar el trayecto que fuimos haciendo, las palabras de nuestro profesor Lorenzo cuando dice: "Focalizar la mirada en las formas de intervención del enseñante no implica abandonar la preocupación por otros temas áulicos e institucionales. Implica incorporar una nueva preocupación, nada menos la que da sentido y razón a nuestra tarea, porque no olvidemos que nuestro oficio es ENSEÑAR" (2001:10)

Otra observación que podemos realizar es acerca de la visible dicotomía que los docentes hacen entre: hábitos, límites, aspectos emocionales y la enseñanza, por un lado, y los contenidos conceptuales por otro. Los primeros son jerarquizados por los docentes como el aspecto que marca la esencia misma de la tarea del docente en Educación Inicial; sin embargo, estos no son vistos como constitutivos del proceso de enseñanza. No aparecen -excepto en algún caso- planificaciones que contemplen los aspectos que en el discurso fueron jerarquizados por la mayoría de los docentes. Podría entonces percibirse cierta contradicción, pues la mayoría de los entrevistados solo efectúan planificaciones tendientes a generar aprendizajes intelectuales.

Por lo que venimos estableciendo, la didáctica es vista desde una perspectiva instrumental, en donde lo fundamental es "la forma", el "saber hacer" y muy poco espacio queda para la reflexión como proceso de crecimiento y formación profesional compartido. Esto podría estar explicando el porqué para la mayoría de los entrevistados la didáctica es algo lejano que poco tiene que ver con su trabajo cotidiano. Además, es significativo relacionar esto con la intervención docente que "casi no pudo ser vista" por ellos. Podríamos llegar a concluir que existe una confusión entre la intervención y la buena voluntad. Nosotras sentimos que si los docentes pudieran vislumbrar la reflexión como un camino, las contradicciones podrían comenzar a desvanecerse. Se podría interpretar entonces, que la didáctica tal y como nosotras la definimos en nuestro encuadre conceptual con elementos tales como: transposiciones, configuraciones, contrato didáctico y situación didáctica, no aparecería como el referente de la mayoría de nuestros colegas de Educación Inicial. 
Para concluir, e intentando dar un paso más en la reflexión, es que citamos nuevamente a Feldman cuando dice: "En la actualidad parece que la enseñanza ha perdido prestigio probablemente porque fue emparentada con sus aspectos más directivos. Basado en que "todo lo que se enseña a un niño impide que lo descubra por sí mismo", se asentó progresivamente la noción de que el ideal educativo es "no enseñar" sino hacer que "las cosas salgan de lo niños". Muchos maestros evitan referirse a su tarea como de "enseñanza" o sólo aceptan que es necesaria en situaciones límites. Al mismo tiempo evitan describir su rol como de "enseñantes" y prefieren el de "guías" u "orientadores" y no pocos asumen con algo de culpa que "de vez en cuando no hay más remedio que enseñar" (1999:16/17). En nuestra profesión las acciones que llevamos adelante sin lugar a dudas no son neutras. Es por eso tal vez, que sentimos desde el comienzo de este trabajo la preocupación en indagar sobre las creencias de los docentes (de alguna manera de nosotras mismas) porque justamente lo que ellos piensan, sienten y creen es crucial para comprender la esencia de nuestra tarea, y sobre todo las consecuencias que la misma conlleva.

Sentimos que se abren interrogantes ya que la construcción y la reconstrucción del conocimiento del hecho educativo es un proceso siempre inacabado, abierto al cambio conceptual que desde lo humano, ético y profesional podrán ir encontrando sus respuestas. Sabemos que nosotras hoy no somos las mismas profesionales -y por ende las mismas personas- que comenzaron este trabajo, hay un antes y un después, que tiene que ver con la búsqueda que cada una ha tenido que efectuar, el cuestionamiento y la confrontación con otros colegas que permitieron que una vez más se produjera uno de los milagros más maravillosos de la vida y muy especialmente de nuestra profesión: "el aprendizaje" - "el crecimiento". Somos conscientes de que nuestro trabajo ha sido simplemente una aproximación a un tema que tal vez, podamos con otras investigaciones ir develando aun más, siempre en busca de lo "valioso" en educación.

\section{Notas}

${ }^{1}$ Situando a la enseñanza, como una actividad reflexiva y constructiva desde la propia actividad del docente y de su potencial, en contraposición a posturas tecnicistas.

${ }^{2}$ Haciendo nuestra la conceptualización que Feldman desarrolla, contraponiendo el enfoque tecnicista al instrumental recuperándose así la dimensión práctica de la tarea de enseñar, y la deliberación práctica en marcos colectivos.

${ }^{3}$ Tomamos el término "oficio de enseñar "de conceptos vertidos por Litwin en su trabajo. "El campo de la didáctica: La búsqueda de una nueva agenda".

${ }^{4}$ En un primer momento quisimos conceptualizar esta categoría como "formas de intervención didáctica", pero como los docentes en muchos casos consideran que no deben enseñar, nos pareció inadecuada esta denominación, y fue sustituida por la de formas de intervención docente. 


\section{Bibliografía}

CAMILLONI, A. y otros. 1995. Corrientes didácticas contemporáneas, Buenos Aires: Paidós.

C.E.P. 1992 Programa de Educación Inicial, Montevideo: C.E.P.

CHEVALLARD, Y. 1997. La transposición didáctica. Buenos Aires: Aique.

DAVINI, M. 1995. La formación docente en cuestión: política y pedagogía. Buenos Aires: Paidós.

DÍAZ BARRIGA, A. 1991. Didáctica. Aportes para una polémica. Buenos Aires: Aique.

ELLIOT, J. 1994. La investigación-acción en educación. Madrid: Morata.

ELLIOT, J. 1996. El cambio educativo desde la investigación acción. Madrid: Morata.

FELDMAN, D. 1999. Ayudar a enseñar. Relaciones entre didáctica y enseñanza. Buenos Aires: Aique.

FREIRE, P. 1994. Cartas a quien pretende enseñar. Sao Paulo: Siglo XXI.

GIROUX, H. 1990. Los profesores como intelectuales. Barcelona/Madrid: Paidós/M.E.C.

IMBERNÓN, F. 1997. La formación y el desarrollo profesional del profesorado. Barcelona: Grao.

LITWIN, E. 1997. Las configuraciones didácticas. Buenos Aires: Paidós.

LORENZO, E. 2001. El discurso didáctico en la Didáctica y fuera de la Didáctica. 45 (1):pp7-9 en: Revista Quehacer Educativo.

MEIRIEU, P. 1992. Aprender, sí. Pero ¿cómo? París: Octaedro.

SACRISTAN, G. y PÉREZ GÓMEZ. 1994. Comprender y transformar la enseñanza. Madrid: Morata.

TAYLOR, S.J. y BOGDAN R. 1987. Introducción a los métodos cualitativos de investigación. Barcelona: Paidós.

VÁZQUEZ, A. 1999. En busca de la enseñanza perdida. México: Paidós.

ZABALZA, M. 1996. Calidad en la educación infantil. Madrid: Narcea.

*, ** Diploma en Educación, Universidad ORT Uruguay 